TRANSACTIONS OF THE

AMERICAN MATHEMATICAL SOCIETY

Volume 363, Number 3, March 2011, Pages 1619-1629

S 0002-9947(2010)05196-7

Article electronically published on September 23, 2010

\title{
A TRICHOTOMY OF COUNTABLE, STABLE, UNSUPERSTABLE THEORIES
}

\author{
MICHAEL C. LASKOWSKI AND S. SHELAH
}

\begin{abstract}
Every countable, strictly stable theory either has the Dimensional Order Property (DOP), is deep, or admits an 'abelian group witness to unsuperstability'. To obtain this and other results, we develop the notion of a 'regular ideal' of formulas and study types that are minimal with respect to such an ideal.
\end{abstract}

\section{INTRODUCTION}

By definition, a stable, unsuperstable theory (henceforth called strictly stable) admits a type that is not based on any finite subset of its domain. From this one sees that such a theory admits trees of definable sets. That is, there is a sequence $\left\langle\varphi_{n}(x, y): n \in \omega\right\rangle$ of formulas such that for any cardinal $\kappa$ there are definable sets $\left\{\varphi_{n}\left(x, a_{\nu}\right): \nu \in{ }^{<\omega} \kappa\right\}$ giving rise to $\kappa^{\aleph_{0}}$ partial types $\left\{p_{\mu}: \mu \in{ }^{\omega} \kappa\right\}$ where each $p_{\mu}$ forks over $\left\{a_{\mu \mid k}: k<n\right\}$ for all $n \in \omega$. In [12] the second author used these trees to count the number of uncountable models or to find the maximal size of a family of pairwise nonembeddable models of a fixed cardinality of any stable, unsuperstable theory. However, for other combinatorial questions, such as computing the Karp complexity of the class of uncountable models of such a theory, the existence of these trees does not seem to be sufficient. Here, with Theorem 4.3, we prove that when the language is countable, any strictly stable theory exhibits at least one of the three more detailed nonstructural properties mentioned in the abstract. This theorem is used in 9], but it is likely to be applicable to other contexts as well. Two of the alternatives, the Dimensional Order Property (DOP) or a theory being deep appear in 12 and are compatible with superstability. The third alternative is new and is captured by the following definition:

Definition 1.1. An abelian group witness to unsuperstability is a descending sequence $\left\langle A_{n}: n \in \omega\right\rangle$ of abelian groups with $\left[A_{n}: A_{n+1}\right]$ infinite for each $n$ such that the intersection $A=\bigcap_{n} A_{n}$ is connected and whose generic type is regular.

The existence of such a sequence readily contradicts superstability as for any cardinal $\kappa$, one immediately obtains a family of $\left\{C_{\mu}: \mu \in{ }^{\omega} \kappa\right\}$ of cosets of $A$ indexed by a tree. The family of generic types of these cosets form a counterexample to

Received by the editors September 24, 2008 and, in revised form, September 2, 2009.

2010 Mathematics Subject Classification. Primary 03C45.

The first author was partially supported by NSF grants DMS-0600217 and DMS-0901336.

The second author's research was partially supported by NSF grants DMS 9704477, DMS 0072560, DMS 0100794 and DMS 0600940 and Israel Science Foundation Grant no. 242/03. Publication 871. 
superstability witnessed by regular types. As well, with Theorems 4.3 and 4.4 we see that one can frequently say more about the generic type of $A$. This added information is used in [9].

As examples, the theory of separably closed fields has DOP ([1] or [2]). The theory of infinitely many, refining equivalence relations in which each $E_{n+1}$ splits each $E_{n}$-class into infinitely many classes is strictly stable and deep, as is the model completion of a single unary function. For the third case, the theory of $\left(\mathbb{Z}^{\omega},+, U_{n}\right)_{n \in \omega}$, where each $U_{n}$ is the subgroup in which the first $n$ coordinates are zero, is the paradigm of an abelian group witness to unsuperstability.

In order to establish these theorems, much of the paper discusses the notion of a regular ideal of formulas (see Definition 2.3). The origins of these ideas date back to Section V.4 of [12] and have been reworked and expanded in [3] and [10. As well, the proof of the trichotomy depends on results from $[8$.

Our notation is standard, and complies with either [10] or [12]. For a stable theory $T, \kappa_{r}(T)$ denotes the least regular cardinal $\kappa$ such that there is no forking chain of length $\kappa$. Thus, a stable theory is superstable if and only if $\kappa_{r}(T)=\aleph_{0}$ and $\kappa_{r}(T)=\aleph_{1}$ when $T$ is countable and strictly stable. We call a model 'a-saturated' (a-prime) in place of ' $\mathbf{F}_{\kappa_{r}(T)^{-}}^{a}$-saturated' $\left(\mathbf{F}_{\kappa_{r}(T)}^{a}\right.$-prime).

Throughout the whole of this paper we assume ' $\mathbf{T}=T^{\mathbf{e q}}$ '. That is, $T$ is a stable theory in a multi-sorted language, $\mathfrak{C}$ is a large, saturated model of $T$, and the language $L$ is closed under the following operation: If $E(\bar{x}, \bar{y})$ is a definable equivalence relation, then there is a sort $U_{E}$ and a definable surjection $f_{E}: \mathfrak{C}^{\lg (\bar{x})} \rightarrow$ $U_{E}(\mathfrak{C})$ in the language $L$. In particular, the set of sorts is closed under finite products. Thus any finite tuple of elements from varying sorts can be viewed as an element of the product sort. With this identification, every formula can be considered to have a single free variable. As notation, $L(\mathfrak{C})$ denotes the set of formulas with parameters from $\mathfrak{C}$ and for a specific sort $s, L_{s}(\mathfrak{C})$ denotes the $L(\mathfrak{C})$ formulas $\varphi(x)$ in which the free variable has sort $s$.

\section{Regular ideals}

Definition 2.1. An invariant ideal $\mathcal{I D}$ is a subset of $L(\mathfrak{C})$ containing all algebraic formulas, closed under automorphisms of $\mathfrak{C}$, and for any sort $s$ and any $\varphi, \psi \in L_{s}(\mathfrak{C})$

(1) If $\varphi, \psi \in \mathcal{I D}$, then $\varphi \vee \psi \in \mathcal{I D}$; and

(2) If $\varphi \vdash \psi$ and $\psi \in \mathcal{I D}$, then $\varphi \in \mathcal{I D}$.

A partial type $\Gamma$ (i.e., a subset of $L_{s}(\mathfrak{C})$ for some sort $s$ ) is $\mathcal{I D}$-small if it entails some element of $\mathcal{I D} \cap L_{s}(\mathfrak{C})$.

Examples of invariant ideals include the algebraic formulas, the superstable formulas (see Definition 4.1) or the ideal of formulas with Morley rank. These and other examples are discussed in [10]. Many times we will make use of the fact that formulas in $\mathcal{I D}$ may have 'hidden' parameters.

Lemma 2.2. Let $\mathcal{I D}$ be any invariant ideal.

(1) A complete type $p \in S(A)$ is $\mathcal{I D}$-small if and only if $p \cap \mathcal{I D} \neq \emptyset$.

(2) For any $A$ and $a$, stp $(a / A)$ is $\mathcal{I D}$-small if and only if $t p(a / A)$ is $\mathcal{I D}$-small.

(3) If $A \subseteq B$ and $\operatorname{tp}(a / B)$ does not fork over $A$, then $\operatorname{tp}(a / A)$ is $\mathcal{I D}$-small if and only if $t p(a / B)$ is $\mathcal{I D}$-small. 
Proof. (1) Right to left is immediate. For the converse, assume $p$ entails $\psi \in \mathcal{I D}$. By compactness there is $\varphi \in p$ such that $\varphi \vdash \psi$, hence $\varphi \in \mathcal{I D}$.

(2) Right to left is clear. If $\operatorname{stp}(a / A)$ entails $\psi(x, b) \in \mathcal{I D}$, then by compactness and the finite equivalence relation theorem there is an $A$-definable equivalence relation $E(x, y)$ with finitely many classes such that $\operatorname{tp}(a / A) \cup\{E(x, c)\} \vdash \psi(x, b)$ for some $c$. Choose $A$-automorphisms $\left\{\sigma_{i}: i<n\right\}$ of $\mathfrak{C}$ such that $\left\{E\left(x, \sigma_{i}(c)\right): i<n\right\}$ includes all the $E$-classes. Since $\mathcal{I D}$ is an invariant ideal $\bigvee_{i<n} \psi\left(x, \sigma_{i}(b)\right) \in \mathcal{I D}$ and $\operatorname{tp}(a / A) \vdash \bigvee_{i<n} \psi\left(x, \sigma_{i}(b)\right)$.

(3) By (2) it suffices to prove this for strong types. Assume $\operatorname{stp}(a / B)$ is $\mathcal{I D}$ small. By (1) and (2), choose $\psi(x, b) \in \operatorname{tp}(a / B) \cap \mathcal{I D}$. Choose $\left\{b_{i}: i \in \kappa(T)\right\}$ independent over $A$, each having the same strong type over $A$ as $b$. Since $\mathcal{I D}$ is invariant, $\psi\left(x, b_{i}\right) \in \mathcal{I D}$ for each $i$. Furthermore, since any $a^{\prime}$ realizing $\operatorname{stp}(a / A)$ is independent with some $b_{i}$ over $A, a b$ and $a^{\prime} b_{i}$ realize the same strong type over $A$, hence $\psi\left(a^{\prime}, b_{i}\right)$ holds. By compactness, there is a finite subset $F$ such that $\operatorname{stp}(a / A) \vdash \bigvee_{i \in F} \psi\left(x, b_{i}\right), \operatorname{so} \operatorname{stp}(a / A)$ is $\mathcal{I D}$-small.

Definition 2.3. An invariant ideal $\mathcal{I D}$ is regular if, for all $L(\mathfrak{C})$-formulas $\psi(y)$ and $\theta(x, y)$, if $\psi \in \mathcal{I D}$ and $\theta(x, b) \in \mathcal{I D}$ for every $b \in \psi(\mathfrak{C})$, then the formula $\exists y(\psi(y) \wedge \theta(x, y)) \in \mathcal{I D}$.

By analogy, recall that a cardinal $\kappa$ is regular if and only if the union of fewer than $\kappa$ sets, each of size less than $\kappa$, has size less than $\kappa$.

We call a strong type $\operatorname{stp}(a / A) \mathcal{I D}$-internal if there is a set $B \supseteq A$ independent from $a$ over $A$, a $B$-definable function $f$, and elements $\bar{c}$ such that $\operatorname{tp}(c / B)$ is $\mathcal{I D}$ small for each $c \in \bar{c}$ and $a=f(\bar{c})$. The strong type $\operatorname{stp}(a / A)$ is $\mathcal{I D}$-analyzable if there is a finite sequence $\left\langle a_{i}: i \leq n\right\rangle$ from $\operatorname{dcl}(A a)$ such that $a_{n}=a$ and $\operatorname{stp}\left(a_{i} / A \cup\left\{a_{j}: j<i\right\}\right)$ is $\mathcal{I D}$-internal for each $i \leq n$. Since $\mathcal{I D}$ is a collection of formulas, this definition of analyzability is equivalent to the usual one, see e.g., [10.

In order to iterate the defining property of a regular ideal, we need the following notion, whose terminology is borrowed from 6 .

Definition 2.4. A formula $\varphi(x, c)$ is in $\mathcal{I D}$, provably over $B$ if there is some $\theta(y) \in \operatorname{tp}(c / B)$ such that $\varphi\left(x, c^{\prime}\right) \in \mathcal{I D}$ for every $c^{\prime}$ realizing $\theta$.

Lemma 2.5. For all sets $B$ and every $n \in \omega$, if $\varphi\left(x, y_{0}, \ldots, y_{n-1}\right)$ is $B$-definable and $a, c_{0}, \ldots, c_{n-1}$ satisfy:

(1) $t p\left(c_{i} / B\right)$ is $\mathcal{I D}$-small for each $i<n$;

(2) $\varphi\left(x, c_{0}, \ldots, c_{n-1}\right) \in \mathcal{I D}$ provably over $B$; and

(3) $\varphi\left(a, c_{0}, \ldots, c_{n-1}\right)$,

then $\operatorname{tp}(a / B)$ is $\mathcal{I D}$-small.

Proof. Fix any set $B$. We argue by induction on $n$. If $n=0$ the formula $\varphi(x)$ itself witnesses that $\operatorname{tp}(a / B)$ is $\mathcal{I D}$-small. Assume the result holds for $n$ and fix a formula $\varphi\left(x, c_{0}, \ldots, c_{n}\right)$ and $a, c_{0}, \ldots, c_{n}$ satisfying Conditions (1)-(3). By (1) and Lemma 2.2 choose $\psi\left(y_{n}\right) \in \operatorname{tp}\left(c_{n} / B\right) \cap \mathcal{I D}$. By (2), choose $\theta \in \operatorname{tp}\left(c_{0}, \ldots, c_{n} / B\right)$ such that $\varphi\left(x, c_{0}^{\prime}, \ldots, c_{n}^{\prime}\right) \in \mathcal{I D}$ for all $c_{0}^{\prime}, \ldots, c_{n}^{\prime}$ realizing $\theta$. By shrinking $\theta$ we may assume that $\theta\left(y_{0}, \ldots, y_{n}\right) \vdash \psi\left(y_{n}\right)$.

Let $\theta^{*}:=\exists y_{n} \theta$ and $\varphi^{*}:=\exists y_{n}(\varphi \wedge \theta)$. By our inductive hypothesis, in order to prove that $\operatorname{tp}(a / B)$ is $\mathcal{I D}$-small, it suffices to show that $\varphi^{*}$ and $a, c_{0}, \ldots, c_{n-1}$ satisfy Conditions (1)-(3). Conditions (1) and (3) are immediate. To establish (2), 
we argue that $\theta^{*}$ witnesses that $\varphi^{*}\left(x, c_{0}, \ldots, c_{n-1}\right) \in \mathcal{I D}$ provably over $B$. Visibly, $\theta^{*} \in \operatorname{tp}\left(c_{0}, \ldots, c_{n-1} / B\right)$. Choose $c_{0}^{\prime}, \ldots, c_{n-1}^{\prime}$ realizing $\theta^{*}$. On one hand, since $\psi \in \mathcal{I D}$ and $\theta \vdash \psi, \theta\left(c_{0}^{\prime}, \ldots, c_{n-1}^{\prime}, y_{n}\right) \in \mathcal{I D}$. On the other hand, by our choice of $\theta, \varphi\left(x, c_{0}^{\prime}, \ldots, c_{n-1}^{\prime}, d\right) \in \mathcal{I D}$ for any $d$ such that $\theta\left(c_{0}^{\prime}, \ldots, c_{n-1}^{\prime}, d\right)$ holds. Thus, $\varphi^{*}\left(x, c_{0}^{\prime}, \ldots, c_{n-1}^{\prime}\right) \in \mathcal{I D}$ since $\mathcal{I D}$ is a regular ideal.

Proposition 2.6. If $\operatorname{stp}(a / A)$ is $\mathcal{I D}$-internal, then tp $(a / A)$ is $\mathcal{I D}$-small.

Proof. Choose $B \supseteq A$ independent from $a$ over $A$, a $B$-definable formula $\varphi(x, \bar{y})$, and a tuple of elements $\bar{c}$ such that each $\operatorname{tp}(c / B)$ is $\mathcal{I D}$-small for each $c \in \bar{c}$, $\varphi(a, \bar{c})$ holds, and $\exists^{=1} x \varphi(x, \bar{c})$. But the formula $\varphi(x, \bar{c}) \in \mathcal{I D}$ provably over $B$ via the formula $\exists^{=1} x \varphi(x, \bar{y})$, so $\operatorname{tp}(a / B)$ is $\mathcal{I D}$-small by Lemma 2.5. That $\operatorname{tp}(a / A)$ is $\mathcal{I D}$-small follows from Lemma 2.2

The reader is cautioned that while $\mathcal{I D}$-internal types are $\mathcal{I D}$-small, this result does not extend to $\mathcal{I D}$-analyzable types. In fact, the theory and type mentioned in Remark 8.1 .6 of [10] gives rise to an example of this. Much of the motivation of this section, and in particular how it differs from treatments in [3] and [10], revolves around how we handle $\mathcal{I D}$-analyzable types that are not $\mathcal{I D}$-small.

Definition 2.7. A strong type $p$ is foreign to $\mathcal{I D}$, written $p \perp \mathcal{I D}$, if $p \perp q$ for every $\mathcal{I D}$-small $q$.

Lemma 2.8. The following are equivalent for any regular ideal $\mathcal{I D}$ and any strong type $p$ :

(1) $p \perp \mathcal{I D}$;

(2) $p \perp q$ for every $\mathcal{I D}$-internal strong type $q$;

(3) $p \perp q$ for every $\mathcal{I D}$-analyzable strong type $q$;

(4) If $p=\operatorname{stp}(a / A)$, then there is no $a^{\prime} \in \operatorname{dcl}(A a)$ such that $t p\left(a^{\prime} / A\right)$ is $\mathcal{I D}$ small.

Proof. (1) $\Rightarrow$ (2) follows immediately from Proposition 2.6 . (2) $\Rightarrow$ (3) follows by induction on the length of the $\mathcal{I D}$-analysis, using the fact that $p \perp \operatorname{tp}(b / B)$ and $p \perp \operatorname{tp}(a / B b)$ implies $p \perp \operatorname{tp}(a b / B) .(3) \Rightarrow(4)$ is trivial, and $(4) \Rightarrow(1)$ follows immediately from (say) Corollary 7.4.6 of [10].

The reader is cautioned that when the regular ideal is not closed under $\mathcal{I D}$ analyzability, the following definitions differ from those in [10].

Definition 2.9. A partial type $\Gamma$ is $\mathcal{I D}$-large if it is not $\mathcal{I D}$-small. $\Gamma$ is $\mathcal{I D}$-minimal if it is $\mathcal{I D}$-large, but any forking extension of $\Gamma$ is $\mathcal{I D}$-small. $\Gamma$ is $\mathcal{I D} \mathcal{D}_{\perp}$-minimal if it is $\mathcal{I D}$-large, but any forking extension $\Gamma \cup\{\theta(x, c)\}$ is $\mathcal{I D}$-small whenever $\operatorname{stp}(c / \operatorname{dom}(\Gamma)) \perp \mathcal{I D}$.

Clearly $\mathcal{I D}$-minimality implies $\mathcal{I D}_{\perp}$-minimality, but one of the applications in Section 4 will use $\mathcal{I} \mathcal{D}_{\perp}$-minimal types that are not $\mathcal{I D}$-minimal.

Lemma 2.10. Let $\mathcal{I D}$ be any regular ideal. If a strong type $p$ is both $\mathcal{I D}_{\perp-\text { minimal }}$ and foreign to $\mathcal{I D}$, then $p$ is regular.

Proof. The point is that a counterexample to the regularity of $p$ can be found within the set of realizations of $p$. If $M$ is a-saturated and $p=\operatorname{tp}(a / M)$ is not regular, then there are a tuple $\bar{c}=\left\langle c_{1}, \ldots, c_{n}\right\rangle$ realizing $p^{(n)}$ for some $n$ and a realization $b$ of $p$ such that $\operatorname{tp}(a / M \bar{c})$ forks over $M, \operatorname{tp}(b / M \bar{c})$ does not fork over $M$, and $\operatorname{tp}(b / M \bar{c} a)$ 
forks over $M \bar{c}$. Let $q=\operatorname{tp}(a / M \bar{c})$ and choose an $L(M)$-formula $\theta(x, \bar{c}) \in q$ such that $p \cup\{\theta(x, \bar{c})\}$ forks over $M$. As $p \perp \mathcal{I D}, p^{(n)} \perp \mathcal{I D}$, so the $\mathcal{I D}_{\perp \text {-minimality of }}$ $p$ implies $\operatorname{tp}(a / M \bar{c})$ is $\mathcal{I D}$-small.

But, since $p$ is foreign to $\mathcal{I D}, \operatorname{tp}(b / M \bar{c})$, which is a nonforking extension of $p$ would be orthogonal to $q$ by Lemma 2.8(2). In particular, $\operatorname{tp}(b / M \bar{c} a)$ would not fork over $M \bar{c}$.

The following easy 'transfer result' will be used in the subsequent sections.

Lemma 2.11. Assume that $B$ is algebraically closed, $p=t p(a / B)$ is foreign to $\mathcal{I D}, q=t p(b / B)$, and $b \in \operatorname{acl}(B a) \backslash B$. Then $q$ is foreign to $\mathcal{I D}$. If, in addition, $p$ is $\mathcal{I D}$-minimal $\left(\mathcal{I D}_{\perp}\right.$-minimal), then $q$ is $\mathcal{I D}$-minimal $\left(\mathcal{I D}_{\perp}\right.$-minimal) as well.

Proof. If $q$ were not foreign to $\mathcal{I D}$, then by Lemma 2.8(4) there is $c \in \operatorname{dcl}(B b) \backslash B$ such that $\operatorname{tp}(c / B)$ is $\mathcal{I D}$-small. Since $\operatorname{tp}(c / B)$ is not algebraic it is not orthogonal to $p$, which, via Lemma 2.8 (2), contradicts $p$ being foreign to $\mathcal{I D}$. Thus $q \perp \mathcal{I D}$.

Next, suppose that $p$ is $\mathcal{I D}$-minimal. Since $p \not \perp q$ and $p \perp \mathcal{I D}, q$ cannot be $\mathcal{I D}$-small. To see that $q$ is $\mathcal{I D}$-minimal, choose $C \supseteq B$ such that $\operatorname{tp}(b / C)$ forks over $B$. Then $\operatorname{tp}(a / C)$ forks over $B$, $\operatorname{sot} \operatorname{tp}(a / C)$ is $\mathcal{I D}$-small. Thus $\operatorname{tp}(b / C)$ is $\mathcal{I D}$-small by Lemma 2.5 .

\section{Chains And Witnessing GRoups}

Throughout this section $\mathcal{I D}$ always denotes a regular ideal.

Definition 3.1. We say $A$ is an $\mathcal{I D}$-subset of $B$, written $A \subseteq_{\mathcal{I D}} B$, if $A \subseteq B$ and $\operatorname{stp}(b / A) \perp \mathcal{I D}$ for every finite tuple $b$ from $B$. When $M$ and $N$ are models we write $M \preceq_{\mathcal{I D}} N$ when both $M \preceq N$ and $M \subseteq_{\mathcal{I D}} N$. A set $A$ is $\mathcal{I D}$-full if $A \subseteq_{\mathcal{I D}} M$ for some (equivalently for every) a-prime model $M$ over $A$.

Lemma 3.2. Let $\mathcal{I D}$ be any regular ideal and assume $M$ is a-saturated.

(1) If $M \preceq N$ are models, then $M \preceq_{\mathcal{I D}} N$ if and only if $\varphi(N)=\varphi(M)$ for all $\varphi \in L(M) \cap \mathcal{I D}$.

(2) If $M \subseteq_{\mathcal{I D}} A$, then $M \preceq_{\mathcal{I D}} M[A]$, where $M[A]$ is any a-prime model over $M \cup A$.

Proof. (1) First suppose $M \preceq_{\mathcal{I D}} N$ and choose $\varphi \in L(M) \cap \mathcal{I D}$. If $c \in \varphi(N)$, then $\operatorname{tp}(c / N)$ is $\mathcal{I D}$-small. If $\operatorname{tp}(c / M)$ were not algebraic, it would be nonorthogonal to an $\mathcal{I D}$-small type, contradicting $\operatorname{tp}(c / M) \perp \mathcal{I D}$. So $\operatorname{tp}(c / M)$ is algebraic, hence $c \in \varphi(M)$. Conversely, if there were $c \in N$ such that $\operatorname{tp}(c / M) \not \perp \mathcal{I D}$, then by Lemma 2.8(4) there is $c^{\prime} \in \operatorname{dcl}(M c) \backslash M$ such that $\operatorname{tp}\left(c^{\prime} / M\right)$ is $\mathcal{I D}$-small. Then $\varphi(N) \neq \varphi(M)$ for any $\varphi \in \operatorname{tp}\left(c^{\prime} / M\right) \cap \mathcal{I D}$.

(2) Recall that because $M$ is a-saturated, $M[A]$ is dominated by $A$ over $M$. Choose any tuple $c$ from $M[A]$. If $\operatorname{tp}(c / M)$ were not foreign to $\mathcal{I D}$, then as $M$ is a-saturated, there is an $\mathcal{I D}$-small type $q \in S(M)$ such that $\operatorname{tp}(c / M) \not \perp q$, hence $\operatorname{tp}(c / M)$ is not almost orthogonal to $q$. Since $c$ is dominated by $A$ over $M$, there is $a$ from $A$ such that $\operatorname{tp}(a / M)$ is not almost orthogonal to $q$, which contradicts $M \subseteq_{\mathcal{I D}} A$.

Definition 3.3. A saturated chain is an elementary chain $\left\langle M_{\alpha}: \alpha<\delta\right\rangle$ of asaturated models in which $M_{\alpha+1}$ realizes every complete type over $M_{\alpha}$ for each $\alpha<\delta$. An $\mathcal{I D}$-chain is a sequence $\left\langle M_{\alpha}: \alpha<\delta\right\rangle$ of a-saturated models such that 
$M_{\alpha} \preceq_{\mathcal{I D}} M_{\beta}$ for all $\alpha<\beta<\delta$ and $M_{\alpha+1}$ realizes every type over $M_{\alpha}$ foreign to $\mathcal{I D}$. A chain (of either kind) is $\mathcal{I D}$-full if the union $\bigcup_{\alpha<\delta} M_{\alpha}$ is an $\mathcal{I D}$-full set.

In general, a saturated chain need not be $\mathcal{I D}$-full. However, if $\mathcal{I D}$ is either the ideal of algebraic formulas or superstable formulas (both of which are regular), then any a-saturated chain is $\mathcal{I D}$-full, since types are based on finite sets. A more complete explanation of this is given in the proof of Lemma 4.2. By contrast, the following Lemma demonstrates that $\mathcal{I D}$-chains are always $\mathcal{I D}$-full.

Lemma 3.4. Every $\mathcal{I D}$-chain is full. That is, if $\left\langle M_{\alpha}: \alpha<\delta\right\rangle$ is an $\mathcal{I D}$-chain, $\delta$ is a nonzero limit ordinal, and $M_{\delta}$ is a-prime over $\bigcup_{\alpha<\delta} M_{\alpha}$, then $M_{\alpha} \preceq_{\mathcal{I D}} M_{\delta}$ for all $\alpha<\delta$.

Proof. By the characterization of $M \preceq_{\mathcal{I D}} N$ given by Lemma $3.2(1)$, the first sentence follows from the second. So fix an $\mathcal{I D}$-chain $\left\langle M_{\alpha}: \alpha<\delta\right\rangle$. Let $N=$ $\bigcup_{\alpha<\delta} M_{\alpha}$ and let $M_{\delta}$ be a-prime over $N$. Fix any $\alpha<\delta$. Since $M_{\alpha} \subseteq_{\mathcal{I D}} M_{\beta}$ for all $\alpha<\beta<\delta, M_{\alpha} \subseteq_{\mathcal{I D}} N$, so $M_{\alpha} \preceq_{\mathcal{I D}} M_{\delta}$ by Lemma 3.2(2).

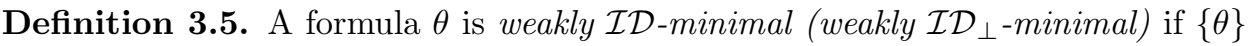
is $\mathcal{I D}$-minimal $\left(\mathcal{I D} \mathcal{D}_{\perp}\right.$-minimal).

We now offer two complementary propositions. The main point of both is that they produce regular types that are 'close' to a given regular ideal. The advantage of (1) is that one obtains $\mathcal{I D}$-minimality at the cost of requiring the chain to be ID -full. In (2) the fullness condition is automatically satisfied by Lemma 3.4, but one only gets $\mathcal{I D} \mathcal{D}_{\perp \text {-minimality. }}$

Proposition 3.6. Fix a regular ideal $\mathcal{I D}$, a countable, stable theory $T$, and an $\mathcal{I D}$-large formula $\varphi$.

(1) Either there is a weakly $\mathcal{I D}$-minimal formula $\psi \vdash \varphi$ or for every $\mathcal{I D}$-full saturated chain $\left\langle M_{n}: n \in \omega\right\rangle$ with $\varphi \in L\left(M_{0}\right)$, there is an $\aleph_{1}$-isolated, ID-minimal $p \in S\left(\bigcup_{n} M_{n}\right)$ with $\varphi \in p$ and $p \perp \mathcal{I D}$.

(2) Either there is a weakly $\mathcal{I D}_{\perp}$-minimal formula $\psi \vdash \varphi$ or for every $\mathcal{I D}$ chain $\left\langle M_{n}: n \in \omega\right\rangle$ with $\varphi \in L\left(M_{0}\right)$, there is an $\aleph_{1}$-isolated, $\mathcal{I D}_{\perp}$-minimal $p \in S\left(\bigcup_{n} M_{n}\right)$ with $\varphi \in p$ and $p \perp \mathcal{I D}$.

Moreover, in either of the two 'second cases' the type $p$ is regular.

Proof. (1) Assume that there is no weakly $\mathcal{I D}$-minimal $\psi \vdash \varphi$. Fix an $\mathcal{I D}$-full saturated chain $\left\langle M_{n}: n \in \omega\right\rangle$ with $\varphi \in L\left(M_{0}\right)$, let $N=\bigcup_{n \in \omega} M_{n}$, and let $M_{\omega}$ be $\aleph_{1}$-prime over $N$. Let $\Delta_{0} \subseteq \Delta_{1} \subseteq \ldots$ be finite sets of formulas with $L=\bigcup_{n \in \omega} \Delta_{n}$. We inductively construct a sequence $\left\langle\varphi_{n}: n \in \omega\right\rangle$ of $\mathcal{I D}$-large formulas as follows: Let $\varphi_{0}$ be our given $\varphi$. Given $\varphi_{n} \vdash \varphi_{0}$ that is an $\mathcal{I D}$-large $L\left(M_{n}\right)$-formula

$$
A_{n}=\left\{\psi \in L\left(M_{n+1}\right): \psi \vdash \varphi_{n}, \psi \text { is } \mathcal{I D} \text {-large and forks over } M_{n}\right\} .
$$

As $M_{n+1}$ realizes every type over $M_{n}$ foreign to $\mathcal{I D}$ and $\varphi_{n}$ is not weakly $\mathcal{I D}$ minimal, $A_{n}$ is nonempty. Choose $\varphi_{n+1} \in A_{n}$ so as to minimize $R\left(\psi, \Delta_{n}, 2\right)$. Let $\Gamma=\left\{\varphi_{n}: n \in \omega\right\}$. We first argue that $\Gamma$ has a unique extension to a complete type in $S(N)$.

Claim. $\Gamma \vdash \neg \psi(x, b)$ for all $\psi(x, b) \in \mathcal{I D} \cap L(N)$.

Proof. If the Claim were to fail, then $\Gamma \cup\{\psi(x, b)\}$ would be consistent, hence would be realized in $M_{\omega}$, say by an element $c$. As the chain is $\mathcal{I D}$-full, $c \in N$. For any $n$ 
such that $c \in M_{n}, \varphi_{n+1}$ was chosen to fork over $M_{n}$, yet is realized in $M_{n}$, which is impossible.

Now let $\psi(x, b)$ be any $L(N)$-formula. Choose $n$ such that $\psi(x, y) \in \Delta_{n}$. As $\varphi_{n+1}$ was chosen to be of minimal $\mathrm{R}\left(-, \Delta_{n}, 2\right)$-rank, it is not possible for both $\varphi_{n+1} \wedge \psi(x, b)$ and $\varphi_{n+1} \wedge \neg \psi(x, b)$ to be in $A_{n}$. For definiteness, suppose $\varphi_{n+1} \wedge$ $\psi(x, b) \notin A_{n}$. Since $\varphi_{n+1}$ forks over $M_{n}$, it must be that $\varphi_{n+1} \wedge \psi(x, b) \in \mathcal{I D}$. Since $\varphi_{n+1} \in \Gamma$, the Claim implies that $\Gamma \vdash \neg \psi(x, b)$. Thus $\Gamma$ implies a complete type in $S(N)$, which we call $p$.

By construction $p$ is $\aleph_{1}$-isolated and is $\mathcal{I D}$-large by the Claim. Since $M_{\omega}$ is $\aleph_{1}$-saturated and $p$ is $\aleph_{1}$-isolated, there is a realization $c$ of $p$ in $M_{\omega}$. If $p$ were not foreign to $\mathcal{I D}$, then by Lemma 2.8 (4) there would be $c^{\prime} \in \operatorname{dcl}(N c) \backslash N$ with $c^{\prime} / N$ $\mathcal{I D}$-small, directly contradicting $\mathcal{I D}$-fullness.

It remains to show that any forking extension of $p$ is $\mathcal{I D}$-small. Let $\theta\left(x, a^{*}\right)$ be any $L(\mathfrak{C})$-formula such that $p \cup \theta\left(x, a^{*}\right)$ forks over $N$. Then for some $n, \theta(x, y) \in \Delta_{n}$ and $\varphi_{n+1} \wedge \theta\left(x, a^{*}\right)$ forks over $N$. Thus, $R\left(\varphi_{n+1} \wedge \theta\left(x, a^{*}\right), \Delta_{n}, 2\right)<R\left(\varphi_{n+1}, \Delta_{n}, 2\right)$. Let $e$ list the parameters occurring in $\varphi_{n+1}$. Since $M_{n+1}$ is saturated, choose $a^{\prime} \in$ $M_{n+1}$ such that $\operatorname{tp}\left(e a^{\prime}\right)=\operatorname{tp}\left(e a^{*}\right)$. It follows from the minimality of $R\left(\varphi_{n+1}, \Delta_{n}, 2\right)$ that $\varphi_{n+1} \wedge \theta\left(x, a^{\prime}\right) \notin A_{n}$, hence $\varphi_{n+1} \wedge \theta\left(x, a^{\prime}\right) \in \mathcal{I D}$. Thus, $\varphi_{n+1} \wedge \theta\left(x, a^{*}\right) \in \mathcal{I D}$ by the invariance of $\mathcal{I D}$.

As for (2) assume that there is no $\mathcal{I D}_{\perp}$-minimal formula implying $\varphi$. Choose an $\mathcal{I D}$-chain $\left\langle M_{n}: n \in \omega\right\rangle$, which is automatically $\mathcal{I D}$-full by Lemma 3.4 The construction of $\Gamma$ and $p$ are analogous, taking $A_{n}^{\prime}=\left\{\psi(x, b) \in A_{n}: \operatorname{tp}\left(b / M_{n}\right) \perp\right.$ $\mathcal{I D}\}$ in place of $A_{n}$ at each step. All that is affected is the final paragraph. As we only need to establish $\mathcal{I} \mathcal{D}_{\perp}$-minimality, choose a formula $\theta\left(x, a^{*}\right)$ with $\operatorname{tp}\left(a^{*} / N\right) \perp$ $\mathcal{I D}$. Choose $n$ as above such that, in addition, $\operatorname{tp}\left(a^{*} / N\right)$ is based and stationary on some finite $b \in M_{n}$. Choose $a^{\prime} \in M_{n+1}$ such that $\operatorname{tp}\left(b e a^{\prime}\right)=\operatorname{tp}\left(b e a^{*}\right)$ and continue as above.

In both cases, the regularity of $p$ follows immediately from Lemma 2.10 .

Recall that a stable theory has $N D I D I P$ if for every elementary chain $\left\langle M_{n}\right.$ : $n \in \omega\rangle$ of models, every type that is nonorthogonal to some a-prime model over $\bigcup_{n \in \omega} M_{n}$ is nonorthogonal to some $M_{n}$. Relationships between NDIDIP and NDOP are explored in 8 .

Proposition 3.7. Fix a countable, stable theory $T$ with NDIDIP and a regular ideal $\mathcal{I D}$ such that the formula ' $x=x$ ' $\notin \mathcal{I D}$.

(1) If there is an an $\mathcal{I D}$-full, saturated chain $\left\langle M_{n}: n \in \omega\right\rangle$, but there is no weakly $\mathcal{I D}$-minimal formula, then there is an abelian group witness to unsuperstability, where in addition the generic type of the intersection is both $\mathcal{I D}$-minimal and foreign to $\mathcal{I D}$.

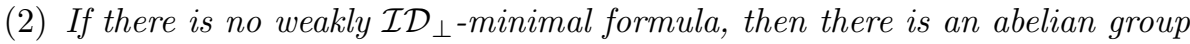
witness to unsuperstability where the generic type of the intersection is

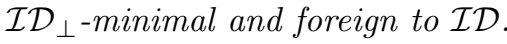

Proof. (1) Fix an $\mathcal{I D}$-full, saturated chain $\left\langle M_{n}: n \in \omega\right\rangle$ and let $N=\bigcup_{n \in \omega} M_{n}$. Using Proposition 3.6 (1) choose $p \in S(N)$ to be $\aleph_{1}$-isolated, foreign to $\mathcal{I} \mathcal{D}$, and $\mathcal{I D}$-minimal, hence regular. Since $T$ has NDIDIP, $p \not \perp M_{n}$. Since $p$ is regular and $M_{n}$ is a-saturated, by Claim X 1.4 of 12 there is a regular type $r_{0} \in S\left(M_{n}\right)$ nonorthogonal to $p$. Let $r$ denote the nonforking extension of $r_{0}$ to $N$. As $p$ and $r$ 
are nonorthogonal there is an integer $m$ such that $p^{(m)}$ is not almost orthogonal to $r^{(\omega)}$. Since $p$ is $\aleph_{1}$-isolated and $M_{n}$ is a-saturated, $N a$ is dominated by $N$ over $M_{n}$ for any $a$ realizing $p$. Thus $p^{(1)}$ is not almost orthogonal to $r^{(\omega)}$ over $N$. Choose $k \geq 1$ maximal such that $p^{(k)}$ is almost orthogonal to $r^{(\omega)}$ over $N$ and choose $\bar{c}$ realizing $p^{(k)}$. Let $B=\operatorname{acl}(N \bar{c})$ and choose a realization $a$ of the nonforking extension of $p$ to $B$.

By Theorem 1 of 4 , there is $b \in \operatorname{dcl}(B a) \backslash B$ and a type-definable, connected group $A$ with a regular generic type $q$ (so $A$ is abelian by Poizat's theorem [11]) and a definable regular, transitive action of $A$ on $p_{1}(\mathfrak{C})$, where $p_{1}=\operatorname{tp}(b / B)$. By Lemma 2.11 the type $p_{1}$ and hence $q$ are both foreign to $\mathcal{I D}$ and $\mathcal{I D}$-minimal. By Theorem 2 of [5] there is a definable supergroup $A_{0} \supseteq A$. By an easy compactness argument we may assume $A_{0}$ is abelian as well. Furthermore, by iterating Theorem 2 of [5] we obtain a descending sequence $\left\langle A_{n}: n \in \omega\right\rangle$ of subgroups of $A_{0}$ with $A=\bigcap_{n \in \omega} A_{n}$.

Thus far we have not guaranteed that $A_{n+1}$ has infinite index in $A_{n}$. In order to show that there is a subsequence of the $A_{n}$ 's with this property and thereby complete the proof of the Proposition, it suffices to prove the following claim:

Claim. For every $n \in \omega$ there is $m \geq n$ such that $\left[A_{n}: A_{m}\right]$ is infinite.

Proof. By symmetry it suffices to show this for $n=0$. Assume that this were not the case, i.e., that $\left[A_{0}, A_{m}\right]$ is finite for each $m$. Then $A$ has bounded index in $A_{0}$. We will obtain a contradiction by showing that the definable set $A_{0}$ is weakly $\mathcal{I D}$-minimal. First, since $q$ is $\mathcal{I D}$-large, the formula defining $A_{0}$ is $\mathcal{I D}$-large as well. Let $\varphi(x, e)$ be any forking extension of the formula defining $A_{0}$ and let $E \subseteq A_{0}$ be the set of realizations of $\varphi(x, e)$. Let $\left\{C_{i}: i<2^{\kappa} \leq 2^{\aleph_{0}}\right\}$ enumerate the cosets of $A$ contained in $A_{0}$. For each $i, E \cap C_{i}$ is a forking extension of $C_{i}$. Since every $C_{i}$ is a translate of $A$ whose generic type is $\mathcal{I D}$-minimal, this implies that $E \cap C_{i}$ is $\mathcal{I D}$-small for each $i$. Hence, $\varphi(x, e) \in \mathcal{I D}$ by compactness (and the fact that $\mathcal{I D}$ is an ideal). Thus, the formula defining $A_{0}$ is weakly $\mathcal{I D}$-minimal, a contradiction.

The proof of (2) is identical, choosing an $\mathcal{I D}$-chain satisfying the hypotheses and using Proposition 3.6(2) in place of 3.6(1).

\section{Applichtions}

Our first application gives a 'trichotomy' for strictly stable theories in a countable language. It uses the ideal of superstable formulas.

Definition 4.1. $\mathbf{R}^{\infty}$ denotes the ideal of superstable formulas (i.e., all formulas $\varphi$ with $\left.R^{\infty}(\varphi)<\infty\right)$.

Equivalently, $\varphi \in \mathbf{R}^{\infty}$ if and only if for all cardinals $\kappa \geq 2^{|T|}$, for any model $M$ of size $\kappa$ containing the parameters of $\varphi$, there are at most $\kappa$ complete types over $M$ extending $\varphi$. In a sense, the following Lemma generalizes the fact that in a superstable theory, any union of a chain of a-saturated models is a-saturated.

Lemma 4.2. $\mathbf{R}^{\infty}$ is a regular ideal, any elementary chain $\left\langle M_{n}: n \in \omega\right\rangle$ of asaturated models is $\mathbf{R}^{\infty}$-full, and there are no weakly $\mathbf{R}^{\infty}$-minimal formulas.

Proof. Invariance under automorphisms of $\mathfrak{C}$ is clear and $\mathbf{R}^{\infty}$ being an ideal follows by counting types. To show regularity, choose $\psi(y) \in \mathbf{R}^{\infty}$ and $\theta(x, y) \in L(\mathfrak{C})$ such that $\theta(x, b) \in \mathbf{R}^{\infty}$ for every $b$ realizing $\psi$. Choose $\kappa \geq 2^{|T|}$ and a model $M$ of size $\kappa$ 
containing the hidden parameters of both $\psi$ and $\theta$. Then there are at most $\kappa$ types $p(x, y) \in S(M)$ extending $\theta(x, y) \wedge \psi(y)$, so the projection $\exists y(\theta(x, y) \wedge \psi(y)) \in \mathbf{R}^{\infty}$ as only $\kappa$ types $q(x) \in S(M)$ extend it.

To establish fullness, fix an elementary chain $\left\langle M_{n}: n \in \omega\right\rangle$ of a-saturated models. Let $N=\bigcup_{n \in \omega} M_{n}$ and choose an a-prime model $M_{\omega}$ over $N$. Because of Lemma 2.8(4), in order to show that $N \subseteq_{\mathcal{I D}} M_{\omega}$ it suffices to show that no element of $c \in M_{\omega} \backslash N$ is $\mathbf{R}^{\infty}$-small. So choose $c \in M_{\omega}$ such that $\operatorname{tp}(c / N)$ is $\mathcal{I D}$-small and we will show that $c \in N$. On one hand, since $\operatorname{tp}(c / N)$ contains a superstable formula, there is a finite $n$ such that $\operatorname{tp}(c / N)$ is based on $M_{n}$. On the other hand, since $M_{\omega}$ is a-prime over $N, \operatorname{tp}(c / N)$ is a-isolated. Thus $\operatorname{tp}\left(c / M_{n}\right)$ is a-isolated as well (see e.g., Theorem IV 4.3(1) of [12]). Since $M_{n}$ is a-saturated, this implies $c \in M_{n} \subseteq N$.

To show that there are no weakly $\mathbf{R}^{\infty}$-minimal formulas, suppose that a formula $\varphi$ has the property that any forking extension of $\varphi$ is $\mathbf{R}^{\infty}$-small. We will show that $\varphi \in \mathbf{R}^{\infty}$ by counting types. Fix a cardinal $\kappa \geq 2^{|T|}$ and a model $M$ of size $\kappa$ containing the parameters of $\varphi$. Let $M_{0} \preceq M$ have size $|T|$ that also contains the parameters containing $\varphi$. It suffices to show that every $p \in S\left(M_{0}\right)$ extending $\varphi$ has at most $\kappa$ extensions to types in $S(M)$. Clearly, there is a unique nonforking extension of $p$ and any forking extension of $p$ contains an $L(M)$-formula witnessing the forking. Each such forking formula $\psi \in \mathbf{R}^{\infty}$, so there are at most $\kappa q \in S(M)$ extending $\psi$. Since the total number of $\psi \in L(M)$ is at most $\kappa, p$ has at most $\kappa$ extensions to types in $S(M)$.

Theorem 4.3. Let $T$ be a stable, unsuperstable theory in a countable language. Then at least one of the following three conditions occurs:

(1) $T$ has the dimensional order property (DOP); or

(2) $T$ has NDOP, but is deep (i.e., there is a sequence $\left\langle M_{n}: n \in \omega\right\rangle$ such that $\operatorname{tp}\left(M_{n+1} / M_{n}\right) \perp M_{n-1}$ for all $\left.n \geq 1\right)$; or

(3) There is an abelian group witness to unsuperstability (see Definition 1.1) in which the generic type of the intersection is both $\mathbf{R}^{\infty}$-minimal and foreign to $\mathbf{R}^{\infty}$.

Proof. To begin, Corollary 1.12 of [8] asserts that any such theory $T$ has NDIDIP. Since $T$ is not superstable the formula ' $x=x^{\prime} \notin \mathbf{R}^{\infty}$. As well, by Lemma 4.2 there are no weakly $\mathbf{R}^{\infty}$-minimal formulas, so Proposition 3.7(1) asserts that an abelian group witness to unsuperstability exists, whose generic type is regular and both $\mathbf{R}^{\infty}$-minimal and foreign to $\mathbf{R}^{\infty}$.

Our second application comes from an attempt to solve the 'Main Gap for $\aleph_{1^{-}}$ saturated models.' As in the previous theorem, the relevant setting is where a countable theory $T$ is stable, unsuperstable, with NDOP, and is shallow. The main open question is whether, for such a theory, every nonalgebraic type $r$ is nonorthogonal to a regular type. The following result sheds some light on this issue. In order to analyze this problem, fix a nonalgebraic, stationary type $r$ over the empty set. Let

$$
\mathcal{I D}_{r}=\{\varphi \in L(\mathfrak{C}): r \perp \varphi\} .
$$

Verifying that $\mathcal{I D}_{r}$ is an invariant ideal is straightforward. To see that it is a regular ideal, fix $L(\mathfrak{C})$-formulas $\psi(y) \in \mathcal{I D}_{r}$ and $\theta(x, y)$ such that $\theta(x, b) \in \mathcal{I D}_{r}$ for every $b$ realizing $\psi$. Choose an a-saturated model $M$ containing the parameters of $\psi$ and $\theta$, pick a realization $c$ of the nonforking extension of $r$ to $M$, and let $M[c]$ 
be any a-prime model over $M c$. To show that $\varphi(x):=\exists y(\theta(x, y) \wedge \psi(y)) \perp r$ it suffices to prove that any realization of $\varphi$ in $M[c]$ is contained in $M$. So choose any $a \in \varphi(M[c])$. Choose $b \in M[c]$ such that $\theta(a, b) \wedge \psi(b)$ holds. Since $r \perp \psi, b \in M$. But then $\theta(x, b)$ is over $M$ and is $\perp r$, so $a \in M$ as well. Thus $\mathcal{I D}_{r}$ is a regular ideal.

Theorem 4.4. Assume that a countable theory $T$ is stable, unsuperstable, has $N D O P$, and is shallow. If a nonalgebraic, stationary type $r$ is orthogonal to every regular type, then there is an abelian group witness to unsuperstability in which the generic type of the intersection $A=\bigcap_{n} A_{n}$ is both $\left(\mathcal{I} \mathcal{D}_{r}\right)_{\perp}$-minimal and foreign to $\mathcal{I D}_{r}$.

Proof. Fix such a type $r$. By naming constants we may assume that $r$ is over the empty set. Note that any formula $\varphi \in r$ is not an element of $\mathcal{I D}_{r}$, so ' $x=x^{\prime} \notin \mathcal{I} \mathcal{D}_{r}$.

Claim. There is no weakly $\left(\mathcal{I} \mathcal{D}_{r}\right)_{\perp}$-minimal formula.

Proof. Assume that $\varphi$ were $\left(\mathcal{I D}_{r}\right)_{\perp}$-minimal. We construct a regular type $p \not \perp r$ as follows: Choose an a-saturated model $M$ containing the parameters in $\varphi$, pick a realization $c$ of the nonforking extension of $r$ to $M$, and choose an a-prime model $M[c]$ over $M c$. Since $\varphi$ is $\mathcal{I D}_{r}$-large we can find an $a \in M[c] \backslash M$ realizing $\varphi$. Choose such an $a$ and let $p=\operatorname{tp}(a / M)$. Clearly, $p \not \perp r$. To see that $p$ is regular, first note that $p$ is $\left(\mathcal{I D}_{r}\right)$-minimal since $p$ is $\mathcal{I D}_{r}$-large and extends $\varphi$. As well, $p$ is foreign to $\mathcal{I D}_{r}$, since if it were not, then by Lemma 2.8(4) there would be $b \in \operatorname{dcl}(M a)$ with $\operatorname{tp}(b / M) \mathcal{I D}_{r}$-small. But then $t p(c / M b)$ would fork over $M$, implying that $r$ is nonorthogonal to an $\mathcal{I D}_{r}$-small type, which is a contradiction. So $p$ is $\left(\mathcal{I D}_{r}\right)$-minimal and foreign to $\mathcal{I D}_{r}$, hence is regular by Lemma 2.10,

The theorem now follows immediately from Proposition $3.6(2)$.

\section{REFERENCES}

[1] Z. Chatzidakis, G. Cherlin, S. Shelah, G. Srour, C. Wood, Orthogonality of types in separably closed fields, Classification theory (Chicago, IL, 1985), 72-88, Lecture Notes in Math., 1292, Springer, Berlin, 1987. MR 1033023 (91f:03074)

[2] F. Delon, Idéaux et types sur les corps séparablement clos [Ideals and types over separably closed fields] Mém. Soc. Math. France (N.S.) no. 33 (1988). MR.986208 (90m:03067)

[3] E. Hrushovski, Kueker's conjecture for stable theories, J. Symbolic Logic 54(1989), no. 1, 207-220. MR987332 (90b:03046)

[4] E. Hrushovski, Almost orthogonal regular types, in Stability in model theory, II (Trento, 1987) Ann. Pure Appl. Logic 45 (1989), no. 2, 139-155. MR1044121 (91k:03083)

[5] E. Hrushovski, Unidimensional theories are superstable, Ann. Pure Appl. Logic 50 (1990), no. 2, 117-138. MR.1081816 (92g:03052)

[6] E. Hrushovski and S. Shelah, A dichotomy theorem for regular types, in Stability in model theory, II (Trento, 1987). Ann. Pure Appl. Logic 45 (1989), no. 2, 157-169. MR 1044122 (91g:03068)

[7] M.C. Laskowski, Descriptive set theory and uncountable model theory, Logic Colloquium '03, 133-145, Lect. Notes Logic, 24, Assoc. Symbol. Logic, La Jolla, CA, 2006. MR2207352 (2006j:03066)

[8] M.C. Laskowski and S. Shelah, Decompositions of saturated models of stable theories, Fund. Math. 191 (2006), no. 2, 95-124. MR2231058 (2007j:03047)

[9] M.C. Laskowski and S. Shelah, The Karp complexity of stable theories, in preparation.

[10] A. Pillay, Geometric Stability Theory, Oxford University Press, 1996. MR 1429864 (98a:03049) 
[11] B. Poizat, Groupes stables, avec types génériques réguliers (French) [Stable groups with regular generic types] Journal of Symbolic Logic 48 (1983), no. 2, 339-355. MR704088 (85e:03082)

[12] S. Shelah, Classification Theory, (revised edition) North Holland, Amsterdam, 1990. MR $1083551(91 \mathrm{k}: 03085)$

Department of Mathematics, University of Maryland, College Park, Maryland 20742

Department of Mathematics, Hebrew University of Jerusalem, 91904 Jerusalem, IsRael - and - Department of Mathematics, Rutgers University, Piscataway, New Jersey 08854 